\title{
The creation and application of a geotechnical block model for an underground mining project
}

\author{
D Sewnun SRK Consulting (South Africa) (Pty) Ltd, South Africa \\ W Joughin SRK Consulting (South Africa) (Pty) Ltd, South Africa \\ M Wanless SRK Consulting (South Africa) (Pty) Ltd, South Africa \\ P Mpunzi SRK Consulting (South Africa) (Pty) Ltd, South Africa
}

\begin{abstract}
The collection and analysis of geotechnical data forms the basis for understanding the geotechnical characteristics and the overall quality of the rock mass in a mining environment. While the use of statistics can provide an impression of the average rock mass quality across a project area, it does not assist with a detailed understanding of the way in which data may be spatially related. With the introduction of geostatistics, the spatial continuity of a dataset may be investigated. This may be carried out with the use of semi-variograms. Once the spatial continuity of a dataset is understood, geostatistical methodologies may be applied to create a geotechnical block model.
\end{abstract}

This paper focuses on the creation of a geotechnical block model which provides a three-dimensional visual representation of rock mass data (in varying levels of confidence) across a project area. This concept is illustrated using a case study where geostatistics is adopted to estimate the rock mass quality across a proposed mining area by applying the appropriate geostatistical methodologies between geotechnical boreholes.

A holistic impression of the rock mass conditions is given by the model, whilst also providing insight on areas where poor rock quality and associated potential instabilities can occur.

This study also brings to light the importance of collecting reliable data during the geotechnical logging process, as the success of any geotechnical block model is highly dependent on the input data that the geostatistics is applied to.

If created with careful consideration it is believed that geotechnical block models are valuable tools which can be continually updated as more data is gathered as mining progresses.

Keywords: geotechnical block modelling, geostatistics, rock mass quality, rock mass classification

\section{Introduction}

A detailed understanding of rock mass conditions is essential for safe, productive mining to take place. To gain insight into the quality of a rock mass, boreholes are usually drilled, geotechnically logged and analysed prior to and during mining operations (Sewnun et al. 2017). During this process, data is often assessed using rock mass classification systems. While the results determined from the use of these systems provide an indication of the rock mass conditions, it can be difficult to form a three-dimensional visual impression of the quality of the rock mass across the mining area. To account for this, spatial variability in rock mass data can be assessed and used to create three-dimensional geotechnical block models. This paper presents a case study where a geotechnical block model has been created for an underground mining project allowing for a three-dimensional visual representative of the rock mass conditions, in which the identification of data-deficient areas and potentially poor ground conditions are outlined. Similar work has also been carried out by Jenkins \& Seymour (2009), Bye (2006a; 2006b), Luke \& Edwards (2004) as well as other authors, which may also be used as a reference point when conducting three-dimensional geotechnical block modelling. 


\section{The underground mining project}

This study was carried out for an underground mining project based on a past-producing high-grade underground copper-zinc mine. The mine is currently investigating the potential to mine two high-grade zinc orebodies (orebody A and orebody B) which will be extracted by longhole stoping. The major lithologies in the mining area are sphalerite (found in the orebody), a folded dolomite formation (comprising of the upper, middle and lower dolomite) and shales, siltstones and sandstones which made up the majority of the hanging wall (Figure 1).

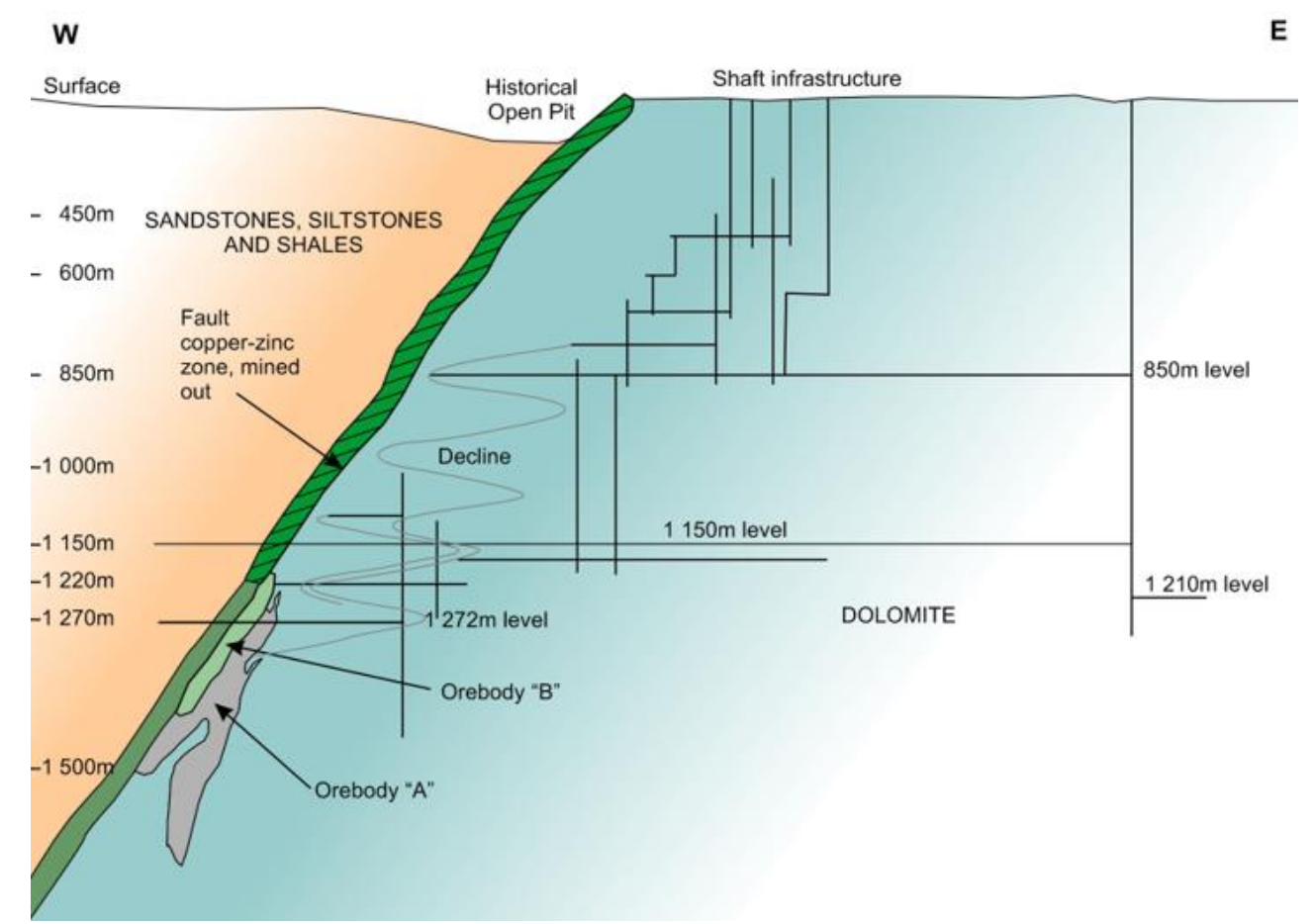

Figure 1 Cross-section showing the major lithologies in the project area

\section{Rock mass quality}

A geotechnical block model has been created for the project based on geotechnical data available from 126 geotechnical borehole logs from boreholes located across the project area (Figure 2). Note, this data includes geotechnical logs from boreholes drilled during each level of study, i.e. Scoping (SS), Pre-feasibility (PFS) and Feasibility (FS). Geotechnical logs used in the SS and PFS are based on the 2014-2015 drilling programme and geotechnical data added to the FS is based on the 2017 drilling programme.

Data input into the geotechnical block model is based on rock mass quality which was determined with the use of a rock mass classification system. To classify the quality of the rock mass, Norwegian Geotechnical Institute's Q-system was applied (Barton et al. 1974). This classification system uses a ' $Q$ ' value to classify the overall rock quality. $Q$ is obtained using the following expression:

$$
Q=\frac{R Q D}{J n} \times \frac{J r}{J a} \times \frac{J w}{S R F}
$$

where:

$$
\begin{array}{llll}
\mathrm{RQD} & =\text { rock quality designation. } & \mathrm{Jn} & =\text { joint set number. } \\
\mathrm{Jr} & =\text { joint roughness number. } & \mathrm{Ja}=\text { joint alteration number. } \\
\mathrm{JW} & =\text { joint water reduction factor. } & \mathrm{SRF} & =\text { stress reduction factor. }
\end{array}
$$


The Q-system was applied to each geotechnical interval for every available borehole.

Note that $Q$ values range between 0.001 and 1,000, whereby a higher $Q$ value indicates better rock quality. As $Q$ values are categorised on irregular intervals, with significantly smaller interval ranges for lower $Q$ values, all $Q$ values calculated were converted from $Q$ to the $\log$ of $Q(\log Q)$ such that it was possible to work within an approximately linear category scale (Table 1 ).

Table 1 Q rock mass quality classification

\begin{tabular}{rrrrrr}
\hline $\mathbf{Q}$ & & logQ & \multicolumn{2}{c}{ Class } \\
\hline 0.001 & 0.01 & -3.0 & -2.0 & Exceptionally poor \\
0.01 & 0.1 & -2.0 & -1.0 & Extremely poor \\
0.1 & 1 & -1.0 & 0.0 & Very poor \\
1 & 4 & 0.0 & 0.6 & Poor \\
4 & 10 & 0.6 & 1.0 & Fair \\
10 & 40 & 1.0 & 1.6 & Good \\
40 & 100 & 1.6 & 2.0 & Very good \\
100 & 400 & 2.0 & 2.6 & Extremely good \\
400 & 1,000 & 2.6 & 3.0 & Exceptionally good \\
\hline
\end{tabular}

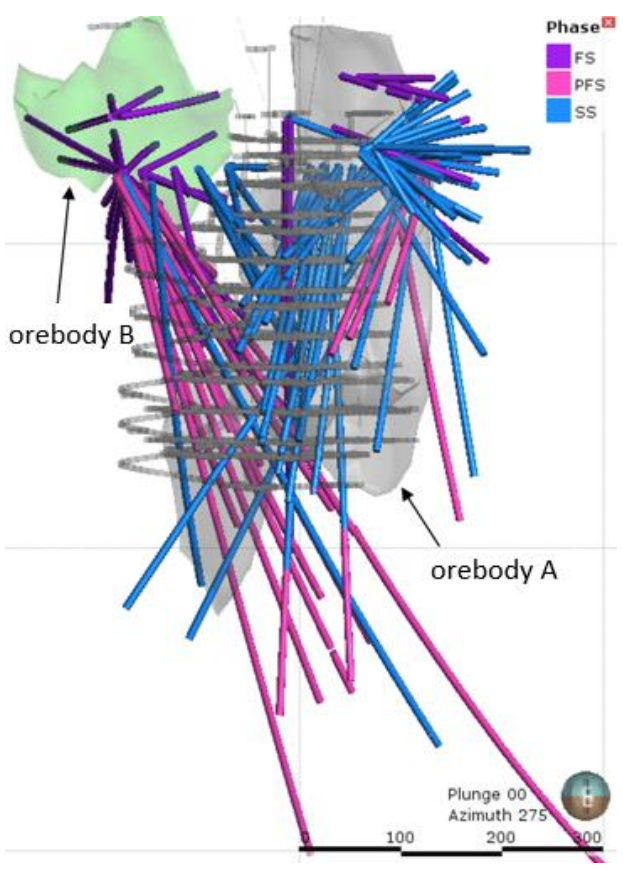

Figure 2 Location of boreholes and orebodies - looking northwest

A histogram illustrating the $\log Q$ rock mass classification results across the project area is presented in Figure 3. Following rock mass classification, a weighted averaging method known as compositing was applied to the data to produce geotechnical intervals of equal lengths, allowing for statistical analysis. This operation was performed using the computer software package LEAPFROG. An interval (compositing) length of $3 \mathrm{~m}$ was chosen for the data as this was the typical core run length. Rock mass classification results based on the composited data are presented in Figure $3 \mathrm{~b}$ and in Figure 4 . The composited logQ values were used to create the geotechnical block model. 


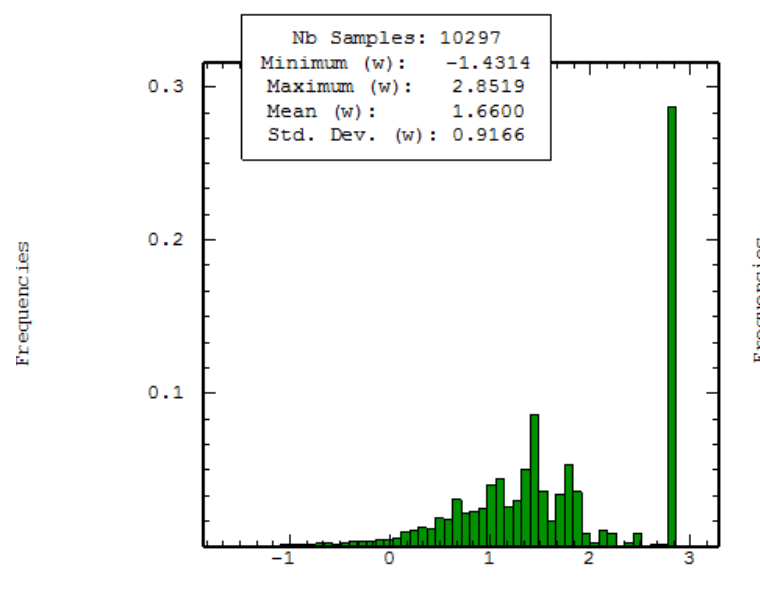

(a)

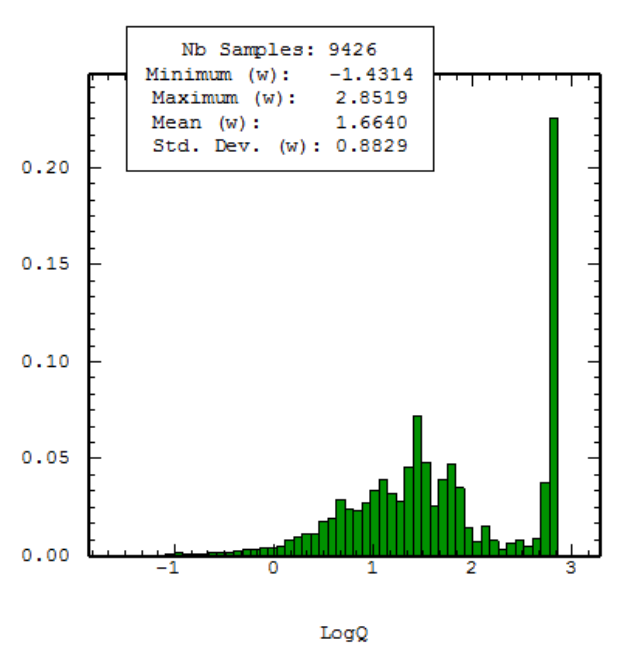

(b)

Figure 3 Histogram of logQ results across project area - (a) Not composited; (b) Composited

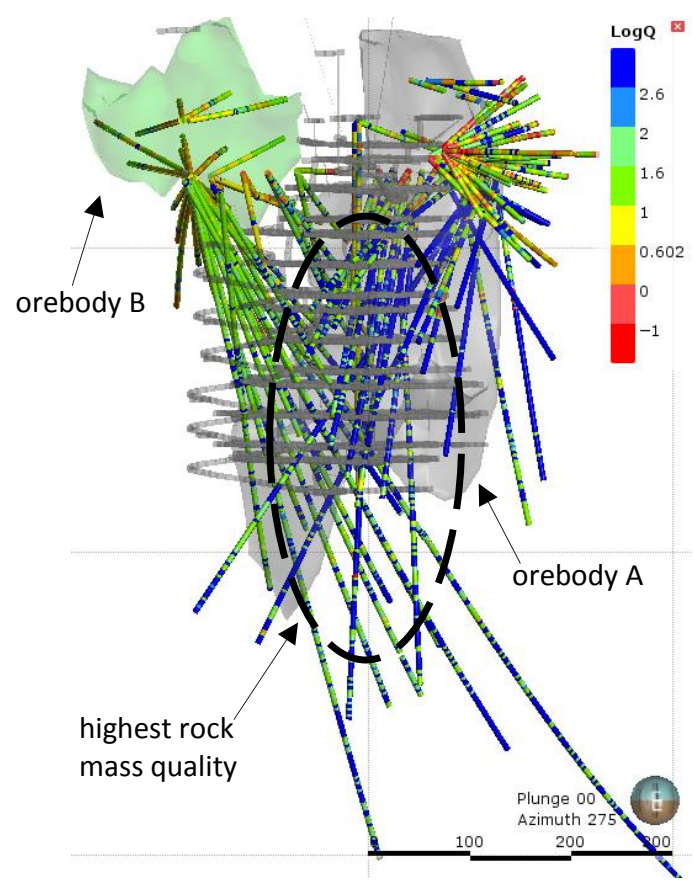

Figure 4 Rock mass classification results across project area - looking northwest

Based on the rock mass classification results it is evident that the rock mass quality is highest in the vicinity of orebody $A$ where the rock mass may be classified as very good to extremely good. The majority of the rock mass in the vicinity of orebody B may be classified as good to very good rock, however there are more localised zones in this area where fair and poor rock quality exists (Figure 4).

\section{$4 \quad$ Geotechnical zones}

On analysis of the rock quality across the project area, it was observed that the rock mass quality is highest in the middle of the project area (where the majority of orebody A is located) compared with the north and south of the project area. It was therefore decided to separate the data into three zones. As the poorer quality rock in the north and south is likely due to the more fractured nature of the upper and lower dolomite, the dolomite boundaries were utilised as a guideline to separate the zones (Figure 5). There is also a presence of chert in the upper dolomite and a greater amount of haematite staining in the lower dolomite which is also associated with the lower rock mass ratings in these areas. 


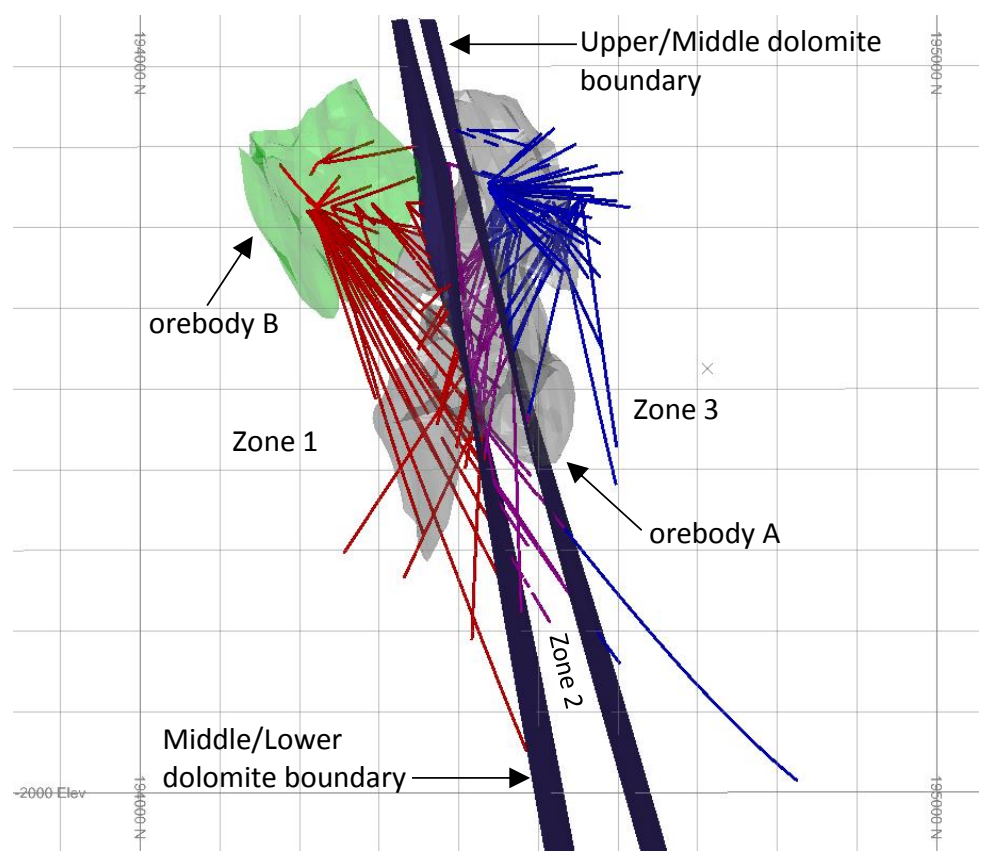

Figure 5 Geotechnical Zones separated by dolomite boundaries (looking northwest)

The distributions of the $\log Q$ values for geotechnical zones 1,2 and 3 are presented in Figure 6 and are summarised in Table 2.
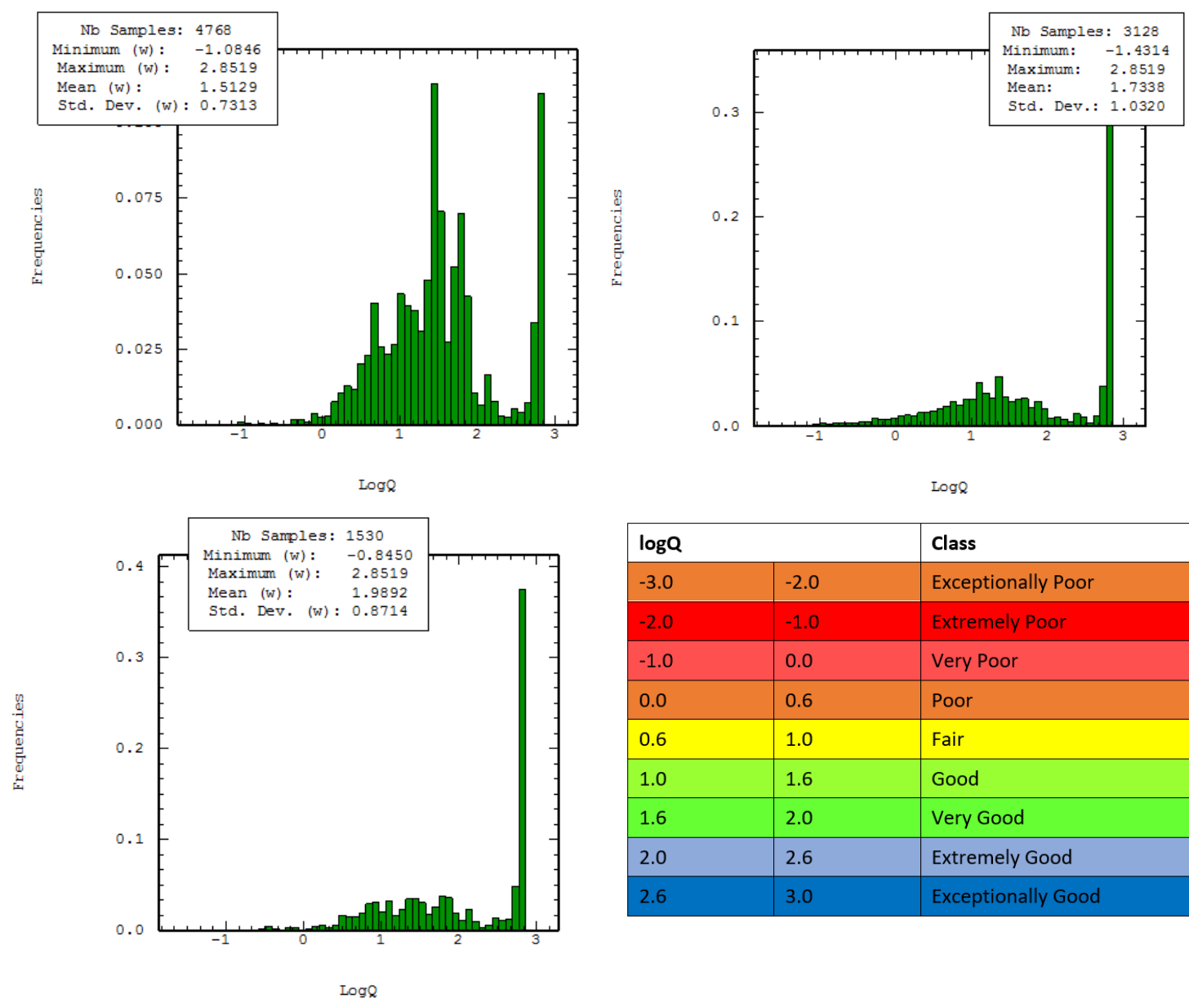

\begin{tabular}{|l|l|l|}
\multicolumn{2}{l}{ Loge } \\
\begin{tabular}{|l|l|l|}
\hline \multicolumn{2}{l}{$\log Q$} & Class \\
\hline-3.0 & -2.0 & Exceptionally Poor \\
\hline-2.0 & -1.0 & Extremely Poor \\
\hline-1.0 & 0.0 & Very Poor \\
\hline 0.0 & 0.6 & Poor \\
\hline 0.6 & 1.0 & Fair \\
\hline 1.0 & 1.6 & Good \\
\hline 1.6 & 2.0 & Very Good \\
\hline 2.0 & 2.6 & Extremely Good \\
\hline 2.6 & 3.0 & Exceptionally Good \\
\hline
\end{tabular}
\end{tabular}

Figure 6 Histograms of logQ per geotechnical zone- Zone 1 (top left) zone 2 (top right) Zone 3 (bottom) 
Table 2 Summary of logQ results

\begin{tabular}{llllll}
\hline Zone & No. of samples & Mean LogQ & Mean Q & Rock mass class \\
\hline Zone 1 (location of orebody B) & 4,768 & 1.51 & 32 & Very good \\
Zone 2 & 1,530 & 1.98 & 96 & Very good \\
Zone 3 & 3,128 & 1.79 & 62 & Very good \\
\hline
\end{tabular}

From the analysis of each geotechnical zone, the following is observed:

- Each zone shares a bimodal negatively skewed distribution of the total dataset. The first grouping ranges within good rock ( $\log Q$ approximately between 1 and 1.5) while the second grouping falls within extremely to exceptionally good rock $(\log Q>2.6)$.

- The mean $\log Q$ value for each zone indicates that the mean quality of the rock is 'good' for zone 1 and 'very good' for zones 2 and 3 (Figure 6 and Table 2).

- The distribution of the results illustrates that there is more good quality rock ( $Q>10$ or $\log Q>0.6)$ in zone 2 (83\%), compared with zone 1 and zone 3 (75\% and 76\%, respectively).

- Furthermore, there is a lower percentage of poor-quality rock $(Q<1$ or $\log Q<0.6)$ in zone $2(6 \%)$, compared with zone 1 and zone 3 (14\% and 9\%).

Geotechnical zones 1, 2 and 3 were thus modelled separately to highlight areas with the poorer quality rock in zones 1 and 3 , and the good to exceptionally good quality rock in zone 2.

\section{$5 \quad$ Comparison of results}

A comparison of the logQ results was carried out between the data collected for the FS level of study and the data used in the PFS (comprises SS and PFS data). This was done to assess any differences in the results between each dataset. The results from this comparison is presented in Figures 7 and 8 .

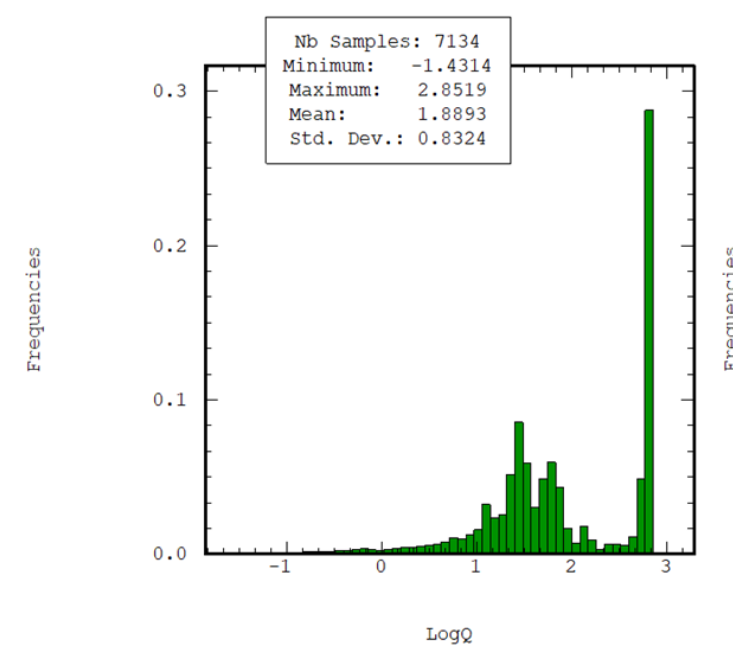

(a)

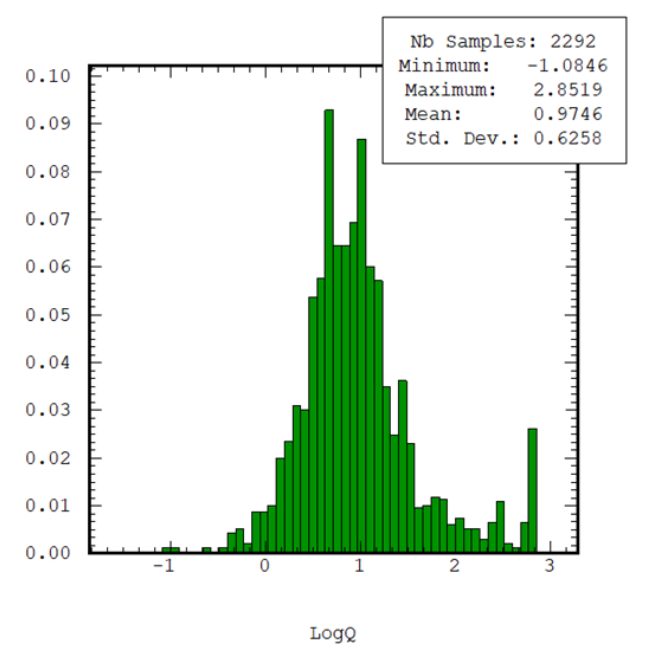

(b)

Figure 7 Histogram of composited logQ results - (a) SS and PFS data; (b) FS data only 

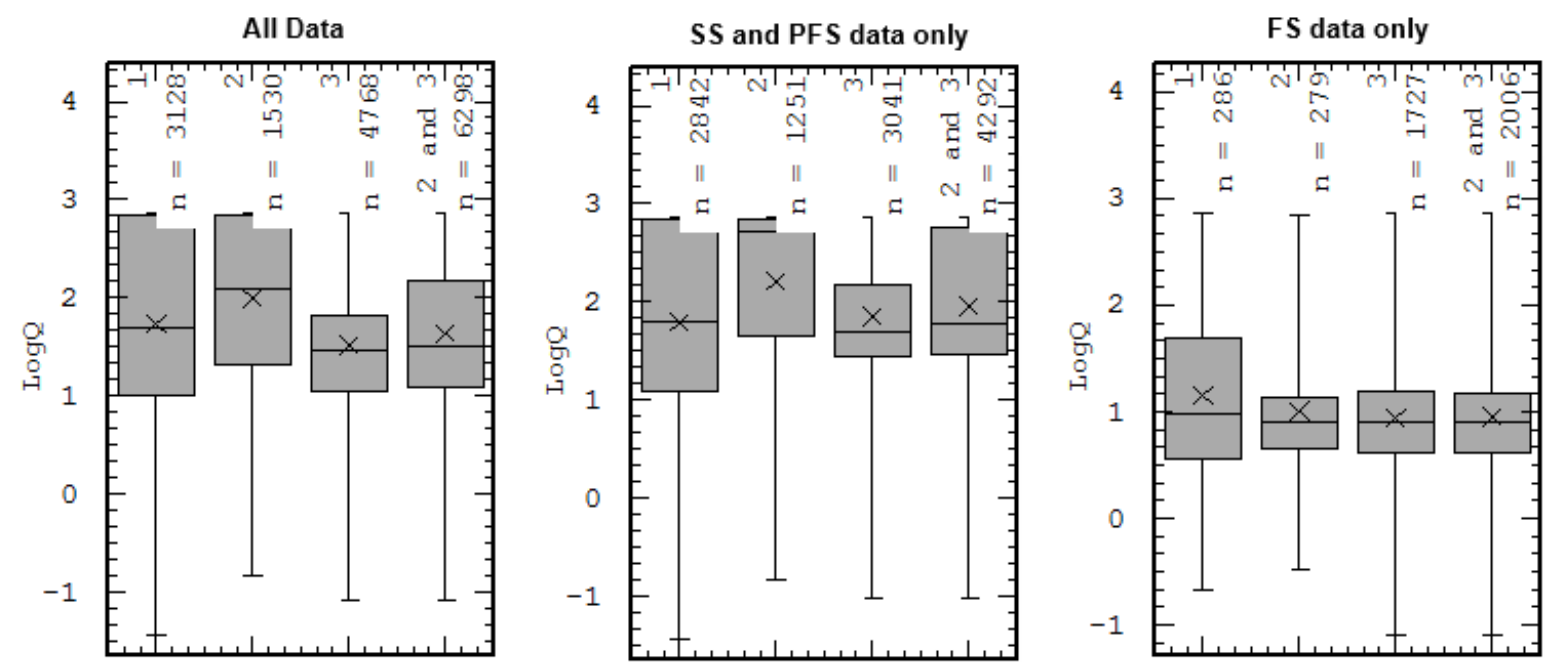

Figure 8 Comparison of logQ results per geotechnical zone, at different levels of study

From the comparison of the results it was observed that the data collected from the additional drilling done for the FS (2017 drilling programme) indicates a lower rock mass quality compared with the data used in the PFS (2014-2015 drilling programme), whereby the mean logQ sits on the border of good rock for the FS data and indicates very good rock for the PFS data (Figures 7 and 8). On interrogation of the data, it was observed that this is due to a combination of the following:

- The majority of the FS holes are located in the lower dolomite region within the vicinity of orebody B (Figure 5), where the rock mass is more fractured and is therefore generally of a lower quality compared with the rock mass present in the vicinity of orebody $A$, which has a very high rock mass quality (Figure 4).

- A new team of mine personnel carried out the geotechnical logging of the 2017 drilling phase holes. As this teams experience lies with geological logging, a more conservative approach was employed compared to the previous phases of drilling. While the standard of logging is acceptable, this teams approach resulted in more conservative logging of each $Q$ parameter resulting in lower $Q$ values compared with the logging conducted by the team during the 2014-2015 drilling programme.

- In many cases red haematite staining was observed in the 2017 drilling phase holes. This was often logged as a containing very thin infill by the 2017 team. On the other hand, this characteristic was generally logged as staining by the 2014/2015 team. This led to higher Ja values and thus lower Q values in the 2017 logs.

Prior to the creation of the block model, minor adjustments were therefore made to the 2017 logs where required (with the aid of core photographs). This included reducing the number of fractures where obvious mechanical breaks were included and updating Ja values from thin infill to staining where it was evident that only staining exists.

\section{Geotechnical model creation}

Datamine Studio RM computer software was used to generate the geotechnical block model. The process followed in creating the model is described below.

\subsection{Semi-variograms}

For the creation of the model, semi-variograms were required and thus created in three orthogonal directions. This was done to gain an impression of the spatial continuity of the data across the project area (Figure 9). 
Zone 1
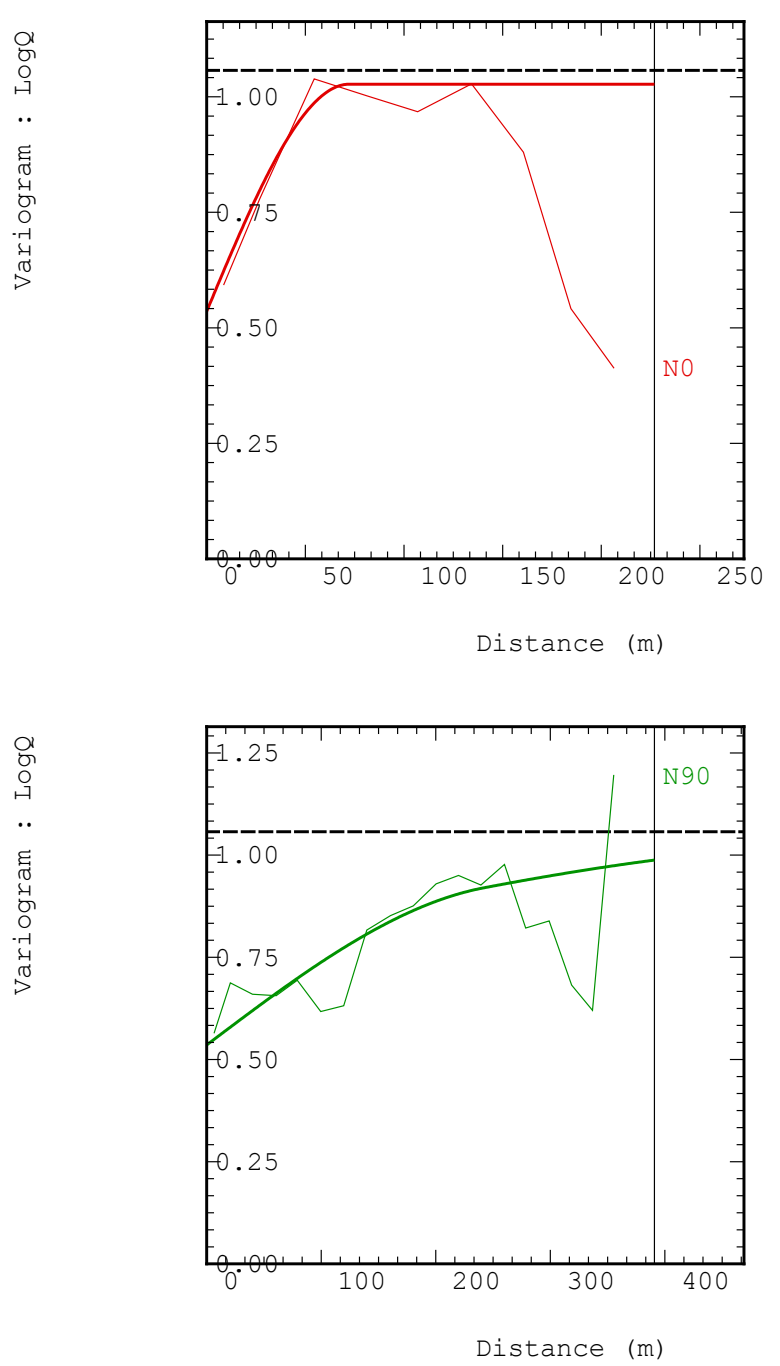

0
0
0
0
1
..
.
5
0
0
0
0
-7
4
0
0

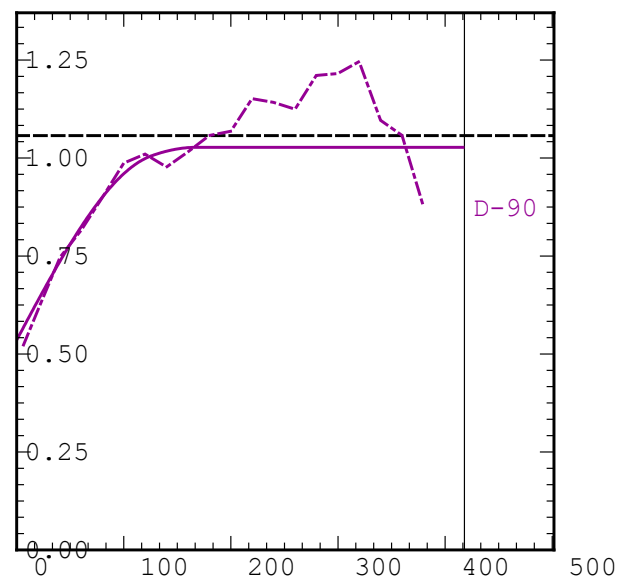

Figure 9 Experimental and modelled semi-variograms
Zone 2 and 3
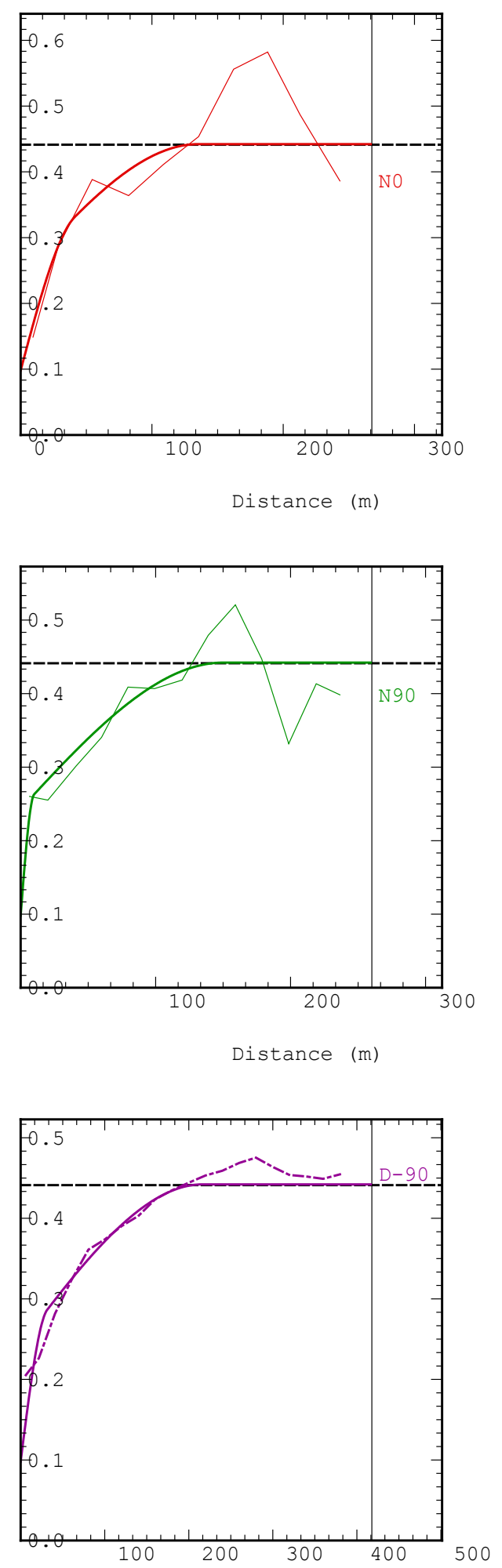
Note from the variograms that zone 2 and 3 were modelled together as there is a lower number of samples in zone 2 (1,530 samples) compared with zone 1 and zone 3 (4,768 and 3,128 samples respectively). The combination of zone 2 and zone 3 thus resulted in an improved variogram which was better suited for use in the creation of the block model.

It was observed in the experimental semi-variograms that zone 1 has the longest range of continuity in an east-west direction, with the shortest range along the north-south axis. In zone 2/3, the semi-variogram structures are similar in all three directions, and this is close to isotropic in behaviour. The semi-variograms also indicate that zone $2 / 3$ has a lower nugget value compared to zone 1 . Although this is the case, practically this only has an impact in the $Y$ axis (north-south) as along the other axes, the first structure has a very short range, and the variance at beyond the first structure $(10-30 \mathrm{~m})$ is equivalent between the two zones aside from the $Y$ axis. Using these results, a two structured spherical semi-variogram model was fitted to the experimental data (Table 3).

Table 3 Semi-Variogram model

\begin{tabular}{lllllllll}
\hline \multirow{2}{*}{ Domain } & Nugget & Sill & \multicolumn{4}{c}{ First structure range } & \multicolumn{3}{c}{ Second structure range } \\
& & & X & Y & Z & X & Y & Z \\
\hline Zone 1 & 0.54 & 0.24 & 72 & 242 & 125 & 72 & 595 & 168 \\
Zone 2/3 & 0.10 & 0.15 & 41 & 10 & 32 & 135 & 150 & 216 \\
\hline
\end{tabular}

\subsection{Prototype}

In addition to the variogram models, a model prototype was required (Table 4) to create the geotechnical block model. The prototype defines the location and dimensions of the block model prior to adding data to the model. A basic model prototype includes the following parameters:

- Model Origin: the corner of the first parent cell i.e. typically the corner of the cell with the lowest $\mathrm{X}, \mathrm{Y}$ and $\mathrm{Z}$ coordinate, referred to as XMORIG, YMORIG, ZMORIG in Datamine.

- Parent Cell: the largest cell allowed in the model, defined by XINC, YINC and ZINC.

- NX, NY, NZ: the number of model parent cells in $X, Y$, and $Z$ directions.

Input parameters for the creation of the model prototype are presented in Table 4.

Table 4 Block model input parameters

\begin{tabular}{cccccccccc}
\hline & \multicolumn{3}{c}{ Model Origin } & \multicolumn{3}{c}{ Parent Cell } & \multicolumn{3}{c}{ Number of Parent Cells } \\
XMORIG & XMORIG & ZMORIG & YINC & ZINC & YINC & NZ & NY & NZ \\
\hline 115700 & 115700 & -1850 & 5 & 5 & 5 & 166 & 120 & 166 \\
\hline
\end{tabular}

\subsection{Statistical approach}

Two methods were employed for the creation of the block model:

1. Nearest neighbour.

2. Ordinary kriging. 
To honour the data within the boreholes, the nearest neighbour method was applied. This method does not involve weighting sample values. Instead, each cell is assigned the value of the 'nearest' sample, where 'nearest' is defined as a transformed or anisotropic distance which takes account of any anisotropy in the spatial distribution of the $\log Q$ values.

Kriging is a geostatistical method for estimating the value of a volume and involves the assignment of weights to the surrounding data. The calculation of the kriged weights is based on the modelled semi-variogram, which describes the correlation between two samples as a function of the distance between them. One of the major advantages of kriging is that the weights are calculated to minimise the error variance. When minimising the error variance, kriging takes into account the spatial location of the samples relative to each another. Hence, if several samples are bunched together, this will be taken into account when the weights are calculated, and the weights reduced accordingly.

There are two commonly applied variations of linear kriging i.e. ordinary kriging and simple kriging. For ordinary kriging, a weight is calculated for each sample, and the sum of these weights is 1 . For simple kriging a weight is calculated for each sample and a weight of $(1-\Sigma W)$ is assigned to the mean. Simple kriging is not as responsive as ordinary kriging to local trends in the data, since it depends partially on the mean, which is assumed to be known, and constant throughout the area. Ordinary kriging is therefore the most commonly used method of kriging and was thus applied to the dataset.

Ordinary kriging was applied to the data using a three-search pass strategy, where the distance from the data was incrementally increased for each search pass (Table 5). This was done to increase the smoothing of the block model as the distance from the data increased, while locally honouring the nearby data. The ranges chosen for each search pass were based on the variogram results. For each search pass, a minimum and maximum number of samples to be utilised was defined. Note that where more than the maximum number of samples within search volume exist, the nearest samples are selected.

Table 5 Search pass parameters

\begin{tabular}{cccc}
\hline Search pass & Range $(\mathbf{m})$ & Minimum no. of samples & Maximum no. of samples \\
\hline 1 & 30 & 6 & 10 \\
2 & 60 & 6 & 12 \\
3 & 90 & 6 & 20 \\
\hline
\end{tabular}

\subsection{Summary of results}

A geotechnical block model was created which indicates the spatial variation in rock mass quality across the project area. This model provides insight on areas where zones of poor ground may exist and therefore allows the opportunity to be made aware of and address potential instabilities. From the block model it was observed that, in general, rock mass conditions within the vicinity of orebody A and orebody B are good. There are however areas where lower rock mass quality is evident. These are generally localised zones in the higher levels of the mine (Figure 11) and in certain areas within the vicinity of orebody $B$.

Sections through the geotechnical block model are presented from Figures 10 to 12 . Figure 13 illustrates the confidence in the block model, which decreases as the distance from the boreholes increase. As there is no data available in the far east of the project area note that this was not modelled. 


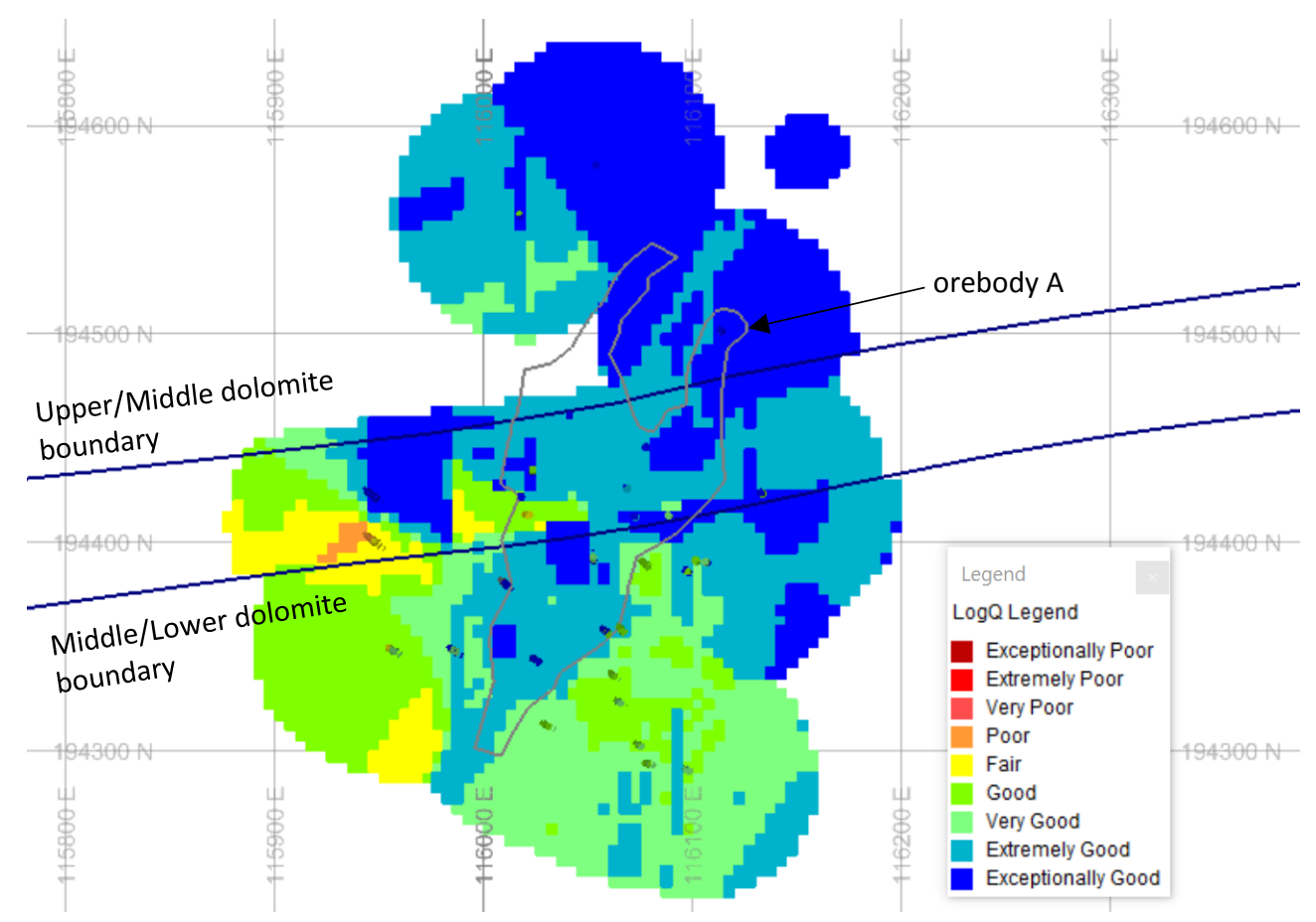

Figure 10 Plan view at 1,495 m

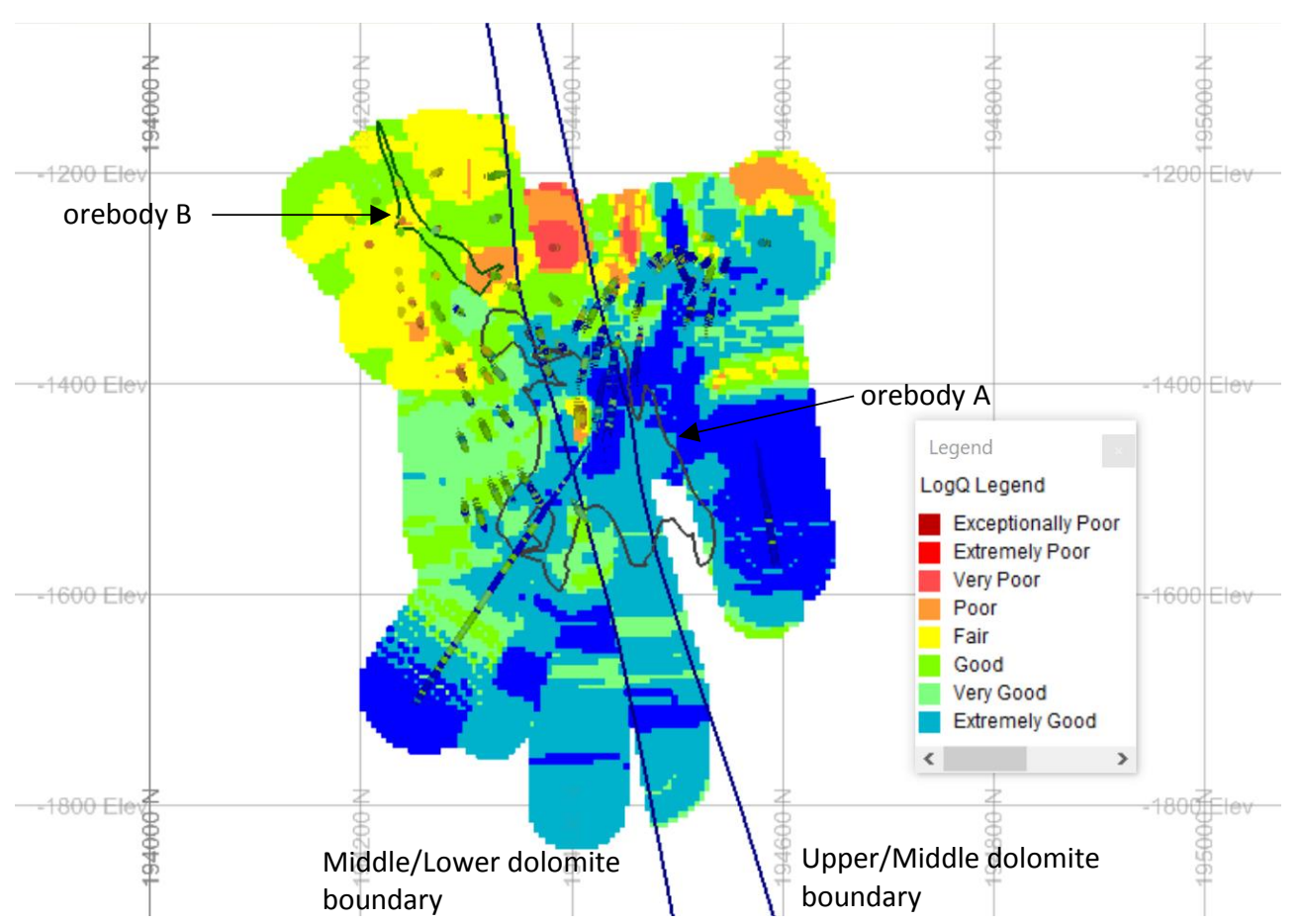

Figure 11 North-south section looking 116054E 


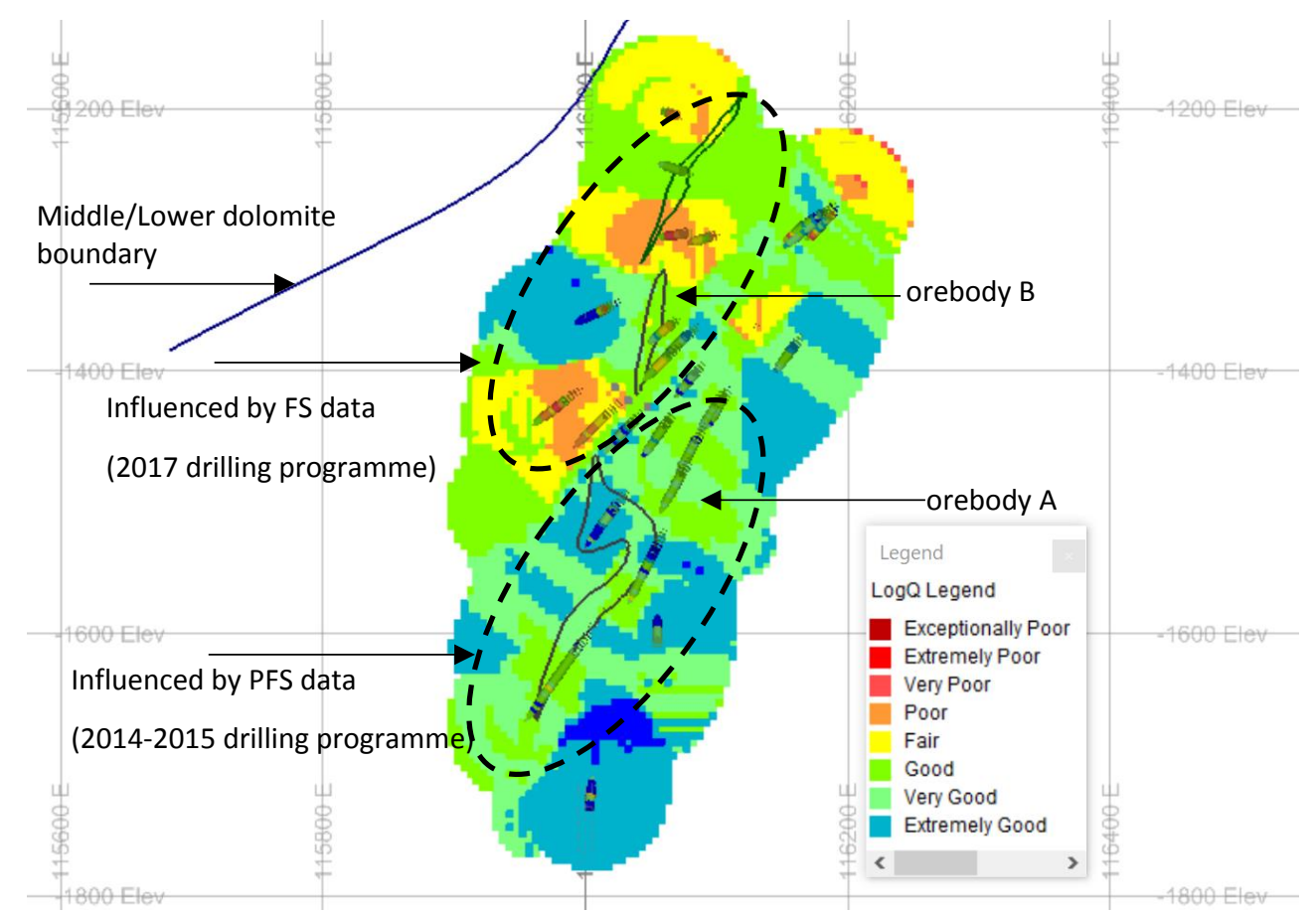

Figure 12 West-east section looking 194325N (Zone 1)

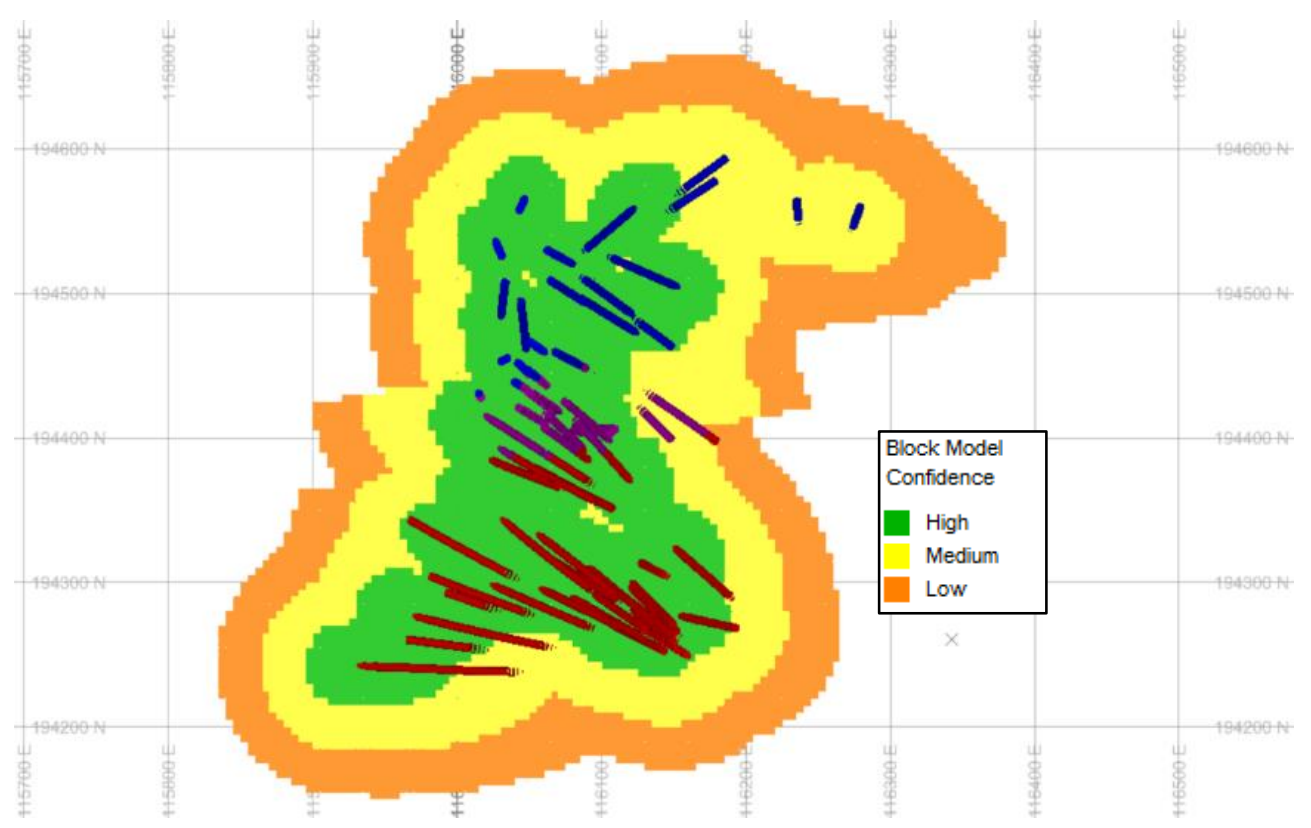

Figure 13 Block model confidence - plan view at 1,390 m

Following an assessment of the block model the following was considered which may be carried out to refine the block model going forward:

- Possible sub-domaining of zone 1 to separate the 2014-2015 drilling programme from the 2017 drilling programme.

- Possible sub-domaining of zone 1, zone 2 and zone 3 to further account for variations in rock mass quality between lithologies.

- Re-assessing the search parameters as follows:

- Aligning the search distances with the anisotropy observed in the semi-variograms. 
- Testing to determine the optimal search distances and number of composites for each search volume.

- Introduce additional factors into the search such as quadrant search rules, and ensuring estimates are not generated from a single borehole only.

- Introducing the use of multiple indicator kriging - treating the LogQ variable as categorical (i.e. using the classes as the variable, rather than the continuous variable) and kriging the indicators of each class. Assess the results and come up with a probability classification for each block, as well as a risk of the value being higher or lower than the preferred probability.

- Trimming the model where estimation was carried out from limited data and extrapolated long distances from the data. This will avoid the introduction of artefacts.

\section{$7 \quad$ Application of the geotechnical block model}

Once the geotechnical block model was created for the project, this model was used to assess the rock mass conditions that may be encountered within the planned developments. This was done by overlaying the block model results and the confidence in the block model onto the developments itself. An example of this is depicted in Figure 14.

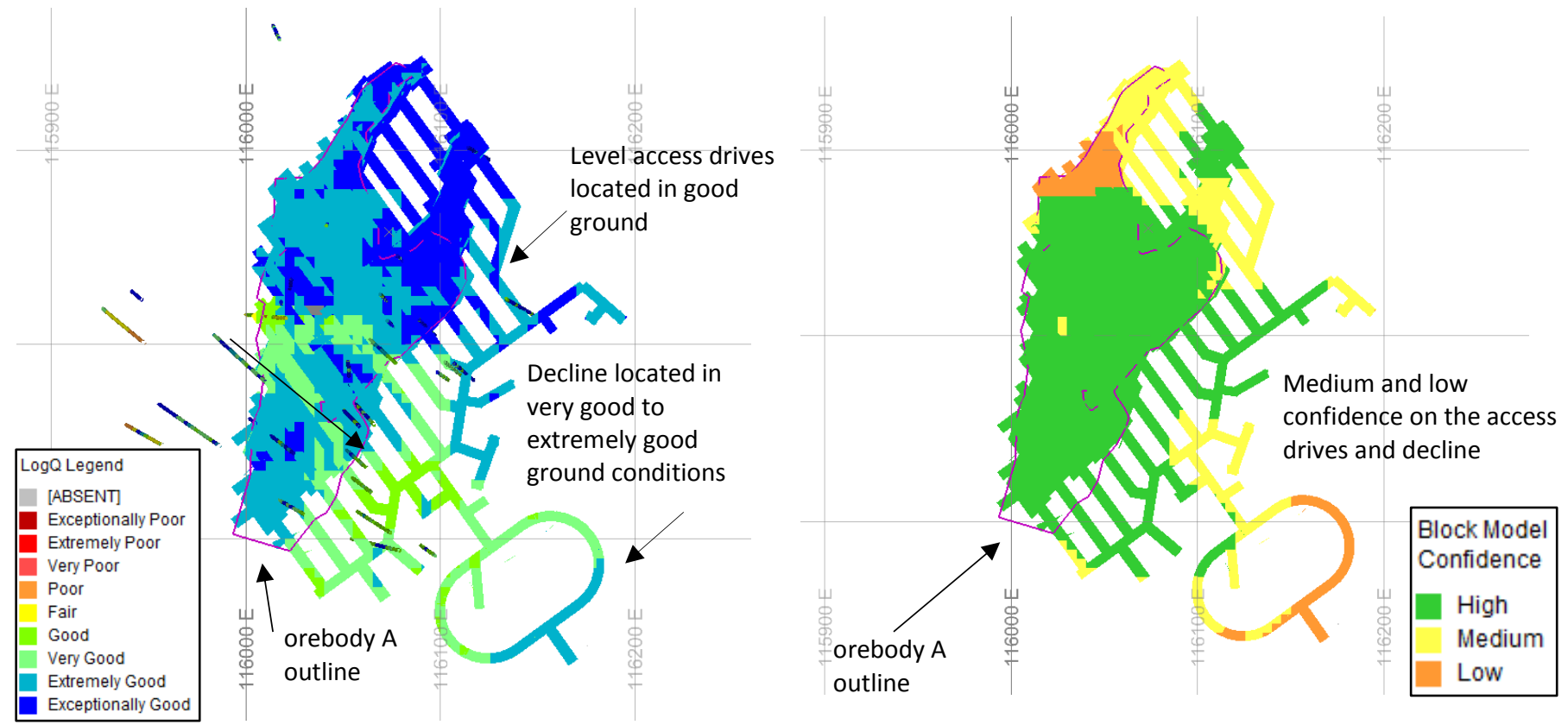

Figure 14 Rock mass quality and block model confidence on level 1515 (plan view)

From Figure 14 it is evident that the rock mass quality is highest in the vicinity of orebody A. In this area the confidence in the block model is also the highest due to a high concentration of boreholes. With the use of the block model rock mass conditions were assessed for each planned mining level.

When applying this block model the following should be kept in mind:

- As the distance from the boreholes increases, the confidence in the block model decreases. The rock mass quality determined is thus the most reliable where there is a high confidence in the model. The geotechnical block model thus serves as a platform which can be built upon on a continuous basis as more data is gathered and as mining takes place.

- While the rock mass quality is generally lower within the vicinity of the orebody B, the FS logging results are also more conservative compared with the SS and PFS logging results. The rock mass quality in the block model in areas where the FS holes are located (typically in the northern area of geotechnical zone 1 where orebody $B$ is located) is therefore more conservative. 


\section{Conclusion}

This study highlights that geotechnical block models may be utilised successfully for various mining applications that require a detailed understanding of the variability in rock mass conditions. Creating such models not only allows for the assessment of the spatial variability in the rock mass information, but in addition allows for the identification of data-deficient and high-risk areas.

Note that while $\log Q$ values were used to represent rock mass quality in this study, geotechnical block models may include other geotechnical parameters such as Mining Rock Mass Ratings (MRMR), fracture frequency (FF), rock quality designation (RQD), etc. These parameters have been modelled in other studies including that by Jenkins \& Seymour (2009) and Bye (2006b), where Datamine was also used for block model creation.

The rock mass does not contain any major geological structures with poor rock mass quality (e.g. geological faults) thus these do not exist within the model. As geological structures may be associated with poor ground conditions, it is important that these are modelled accordingly when present within the vicinity of the mining area. Major faults have been modelled in previous studies such as for the Platreef project (Sewnun et al. 2017).

As geotechnical block models provide an estimation of the rock mass quality in varying levels of confidence across the project area, these models are most appropriate for use when a high level of confidence in the model exists (i.e. where there is an appropriate concentration of data). While this aspect has not been evident in other studies it has been included for the project and is considered a valuable tool. It is therefore believed that every geotechnical block model should contain confidence levels to assist the user accordingly.

Further to the value that may be gained by using geotechnical block models, this study also brings to light the importance of collecting reliable data during the geotechnical logging process, as the success of any geotechnical block model is highly dependent on the input data that the geostatistics is applied to. Data should therefore be carefully assessed prior to use in a model. Furthermore, as geotechnical logging can be subjective it is also imperative that that the logging style of various teams and individuals working on a project are also taken into account as part of this assessment.

If created with careful consideration it is believed that the geotechnical block models are valuable tools which may be continually updated as more data is gathered as mining progresses.

\section{References}

Barton, N, Lien, R \& Lunde, J 1974, 'Engineering classification of rock masses for the design of tunnel support', Rock Mechanics, vol. 6, pp. 189-536.

Bye, A 2006a, 'The application of multi-parametric block models to the mining process', Proceedings of the International Platinum Conference: Platinum Surges Ahead, The Southern African Institute of Mining and Metallurgy, Johannesburg, pp. 259-266.

Bye, A 2006b, 'The strategic and tactical value of a 3D geotechnical model for mining optimization, Anglo Platinum, Sandsloot open pit', The Journal of The South African Institute of Mining and Metallurgy, vol. 106, pp. 97-104.

Jenkins, P \& Seymour, C 2009, 'Mining rock mass models: 3D evaluation of the geotechnical environment for optimal project design and planning', AusIMM Journal, iss. 6.

Luke, DA \& Edwards, A 2004, 'Geotechnical block modelling at BHP Billiton Cannington Mine', in E Villaescusa \& Y Potvin (eds), Proceedings of the Fifth International Symposium on Ground Support, A.A. Balkema, Rotterdam, pp. 129-138.

Sewnun, D, Pillay, O \& Wanless, M 2017, 'Geotechnical block modelling for the 3-dimensional visualisation of rock mass quality in the mining environment', Proceedings of the 9th South African Young Geotechnical Engineers Conference, pp. 1-10. 